\title{
MicroRNA-125b inhibits the proliferation of vascular smooth muscle cells induced by platelet-derived growth factor BB
}

\author{
XIAOGAO WANG ${ }^{*}$, SHIYUAN CHEN ${ }^{*}$, YONG GAO, CHAOWEN YU, \\ ZHONGLIN NIE, RAN LU, YONG SUN and ZEYU GUAN \\ Department of Vascular Surgery, The First Affiliated Hospital of Bengbu Medical College, \\ Bengbu, Anhui 233000, P.R. China
}

Received November 27, 2020; Accepted April 29, 2021

DOI: $10.3892 /$ etm.2021.10223

\begin{abstract}
Excessive proliferation and migration of vascular smooth muscle cells (VSMCs) is the main cause of arteriosclerosis obliterans (ASO). The present study aimed to investigate the role of microRNA (miR)-125b on the proliferation and migration of VSMCs. Platelet-derived grow th factor-BB (PDGF-BB; $20 \mathrm{ng} / \mathrm{ml}$ ) was used to treat VSMCs to establish an in vitro model of ASO. VSMCs were transfected with miR-125b mimic to overexpress miR-125. Cell Counting kit-8 (CCK-8) and BrdU assays were performed to assess the proliferative ability of VSMCs, while Transwell and wound healing assays were performed to assess the migratory ability of VSMCs. Western blot and immunofluorescence analyses were performed to detect the expression levels of angio-associated migratory cell protein (AAMP) and serum response factor (SRF) in VSMCs following transfection with miR-125b mimic or inhibitor. The results demonstrated that miR-125b expression decreased following treatment with PDGF-BB, the effects of which were reversed following transfection with miR-125b mimic. According to the CCK-8 assay, the cell proliferative ability decreased by $\sim 50 \%$ compared with the negative control (NC) group, and $\sim 40 \%$ at day 4 based on the BrdU assay. The results of the Transwell and wound healing assays indicated that the migratory ability of VSMCs significantly decreased in the miR-125b mimic group compared with the NC group. Furthermore, western blot and immunofluorescence analyses demonstrated that AAMP and SRF expression levels decreased following transfection with miR-125b mimic compared with the NC group, the effects of which were reversed following transfection with
\end{abstract}

Correspondence to: Dr Yong Gao, Department of Vascular Surgery, The First Affiliated Hospital of Bengbu Medical College, 2,600 Donghai Road, Bengbu, Anhui 233000, P.R. China E-mail: yonggao03345@163.com

*Contributed equally

Key words: arteriosclerosis obliterans, microRNA-125b, angio-associated migratory cell protein, serum response factor
miR-125 inhibitor. Taken together, the results of the present study suggested that miR-125b inhibits the proliferative and migratory abilities of VSMCs by regulating the expression levels of AAMP and SRF.

\section{Introduction}

Lower extremity arteriosclerosis obliterans (ASO) refers to the deposition of lipids on the blood vessel walls of lower limbs, which is rapidly relieved by interventional therapy (1). However, the 1-year recurrence rate of stenosis is $30-50 \%$ (2). Therefore, it is crucial to identify the molecular mechanisms underlying the ASO process to provide novel strategies for clinical treatments. Excessive proliferation and migration of vascular smooth muscle cells (VSMCs), mainly caused by the monocyte/macrophage-induced inflammatory response, serve pivotal roles in ASO progression and restenosis following treatment (3). Therefore, the prevention and relief of dysfunction of VSMCs are important for ASO treatment.

MicroRNAs (miRNAs/miRs) are involved in the occurrence and progression of ASO. For example, miR-30b-5p is involved in the differentiation of VSMCs through interaction with muscleblind like splicing regulator 1 (4). In addition, miR-143/145 inhibits the proliferation of VSMCs by regulating the expression of mammalian transcription factors and myocardin $(5,6)$. These findings confirm the importance of miRNAs in regulating the dysfunction of VSMCs. The expression of miR-125b is notably altered in ASO (7). For example, a previous study demonstrated that miR-125b expression decreases in VSMCs and miR-125b inhibits the proliferation and migration of VSMCs (7). However, the underlying molecular mechanism of miR-125b in ASO remains unclear. Therefore, it is critical to investigate the potential promoting effects of miR-125b on ASO progression to identify novel treatment strategies for ASO.

The present study aimed to investigate the role of $\mathrm{miR}-125 \mathrm{~b}$ in ASO by simulating ASO with platelet-derived growth factor-BB (PDGF-BB). The effect of PDGF-BB on miR-125b expression was investigated. Subsequently, A10 cells were treated with PDGF-BB to compare the effects of upregulated miR-125b expression on the proliferative and migratory abilities of VSMCs. The potential targets of miR-125b in VSMCs were also investigated. 


\section{Materials and methods}

Cell culture. A10 cells from rat VSMCs were used in the present study. The A10 cell line was purchased from The Cell Bank of Type Culture Collection of The Chinese Academy of Sciences. Cells were maintained in Dulbecco's modified Eagle's medium (DMEM; Gibco; Thermo Fisher Scientific, Inc.) supplemented with $10 \%$ fetal calf serum, $100 \mathrm{U} / \mathrm{ml}$ penicillin and $100 \mathrm{U} / \mathrm{ml}$ streptomycin (Thermo Fisher Scientific, Inc.) at $37^{\circ} \mathrm{C}$ with $5 \% \mathrm{CO}_{2}$. PDGF-BB (20 ng/ml; PeproTech, Inc.), was used to investigate the effect of PDGF-BB on miR-125b expression.

miR-125b mimic and inhibitor transfection. A10 cells were seeded into 6 -well plates $\left(3 \times 10^{5}\right.$ cells per well) for $24 \mathrm{~h}$ and subsequently transfected with miR-125b mimic or miR-125b inhibitor in parallel with their respective control oligos (60 nmol/1; Shanghai GenePharma Co., Ltd.) for $24 \mathrm{~h}$ at $37^{\circ} \mathrm{C}$ using Lipofectamine ${ }^{\circledR} 3000$ reagent (Invitrogen; Thermo Fisher Scientific, Inc.) and Opti-MEM (Thermo Fisher Scientific, Inc.), according to the manufacturer's protocols. The sequences of miR-125b mimic and inhibitor were as follows: miR-125b mimic forward, 5'-UCCCUGAGACCC UAACUUGUGA-3' and reverse, 5'-UCACAAGUUAGG GUCUCAGGGA-3'; NC mimic forward, 5'-UUUGUACUA CACAAAAGUACUG-3' and reverse, 5'-CAGUACUUU UGUGUAGUACAAA-3'; miR-125b inhibitor, 5'-UCACAA GUUAGGGUCUCAGGGA-3' and NC inhibitor, 5'-CAG UACUUUUGUGUAGUACAAA-3'. After RNA interference was performed, the cells were cultured for an additional $48 \mathrm{~h}$. Reverse transcription-quantitative polymerase chain reaction (RT-qPCR) was performed to assess the efficiency of miR-125b transfection.

$R T-q P C R$. Cells with different treatments were collected and total RNA was extracted using TRIzol ${ }^{\circledR}$ reagent (Invitrogen; Thermo Fisher Scientific, Inc.). Total RNA was reverse transcribed into cDNA according to the manufacturer's instructions of the PrimeScript RT Reagent kit (cat. no. RR047A; Takara Biotechnology Co., Ltd.). mRNA expression was analyzed using a $7500 \mathrm{Fast}^{\mathrm{TM}}$ system (Applied Biosystems; Thermo Fisher Scientific, Inc.) and a Sensi Mix SYBR kit (QP10005; OriGene Technologies, Inc.). The PCR conditions were as follows: $95^{\circ} \mathrm{C}$ for $10 \mathrm{sec}, 95^{\circ} \mathrm{C}$ for $5 \mathrm{sec}$ and $60^{\circ} \mathrm{C}$ for $20 \mathrm{sec}(35 \mathrm{cycles})$, followed by $95^{\circ} \mathrm{C}$ for $15 \mathrm{sec}$, and $65^{\circ} \mathrm{C}$ for $10 \mathrm{sec}$. The following primer sequences were used for the qPCR: miR-125b forward, 5'-GCGCTCCCT GAGACCCTAAC-3' and reverse, 5'-TGCAGGGTCCGA GGTAT-3'; and U6 forward, 5'-CTCGCTTCGGCAGCACA-3' and reverse, 5'-AACGCTTCACGAATTTGCGT-3'. Relative expression levels were calculated using the $2^{-\Delta \Delta C t}$ method (8) and normalized to the internal reference gene U6.

Cell proliferation assay. Cell proliferation was assessed via Cell Counting Kit-8 (CCK-8; Beyotime Institute of Biotechnology) and BrdU assays. For the CCK-8 assay, In brief, $2 \times 10^{3}$ cells were seeded into 96 -well plates with $200 \mu \mathrm{l}$ culture medium. Subsequently, $20 \mu \mathrm{l}$ CCK-8 regent was added into each well after $24 \mathrm{~h}$ incubation at $37^{\circ} \mathrm{C}$, and then were incubated at $37^{\circ} \mathrm{C}$ in dark for another $4 \mathrm{~h}$. The optical density (OD) values were detected at $450 \mathrm{~nm}$ using a microplate reader (Tecan Group, Ltd.). Cell proliferation was quantified by standard curves, and a linear standard curve was fitted for log [cell quantity] and OD. For the BrdU assay, cells were seeded into 24 -well plates for $24 \mathrm{~h}$ at $37^{\circ} \mathrm{C}$, followed by different treatments. Cell proliferation was assessed using the BeyoClick ${ }^{\mathrm{TM}}$ EDU-647 system (Beyotime Institute of Biotechnology) at a wavelength of $450 \mathrm{~nm}$.

Transwell assay. For the Transwell assay, $5 \times 10^{4}$ cells with different treatments were plated into the upper chambers (24-well, $8.0 \mu \mathrm{m}$, Corning, Inc.) with serum free medium, and medium supplemented with $10 \%$ FBS was plated into the lower chambers. After $48 \mathrm{~h}$ incubation at $37^{\circ} \mathrm{C}$ with $5 \% \mathrm{CO}_{2}$, the migratory cells were stained with $0.5 \%$ crystal violet for $15 \mathrm{~min}$ at room temperature and observed under a light microscope (magnification, x100; Olympus Corporation).

Wound healing assay. For the wound healing assay, cells were seeded into 6-well plates until they reached $80 \%$ confluence. The cell monolayers were scratched using $200 \mu 1$ pipette tips, washed with PBS to remove the detached cells and cultured in DMEM with $0.5 \%$ FBS for $48 \mathrm{~h}$. The wound distance was observed under a light microscope (magnification, x200; Olympus Corporation).

Western blotting. Cells with different treatments were harvested with a cytology brush, washed with ice-cold PBS and lysed using RIPA lysis buffer (Sigma-Aldrich; Merck $\mathrm{KGaA}$ ), containing a phosphorylase and protease inhibitor mixture (Thermo Fisher Scientific, Inc.). Total protein was quantified via the BCA assay. Proteins (40 $\mu \mathrm{g}$ per lane) were separated by $10 \%$ SDS-PAGE, separated proteins were transferred to PVDF membranes, which were subsequently blocked with Tris-buffered saline with Tween solution $(0.1 \%$ Tween-20) and 5\% skimmed milk at $4^{\circ} \mathrm{C}$ overnight. The membranes were incubated with primary antibodies against SRF (dilution, 1:1,000; cat. no. 16821-1-AP; ProteinTech Group, Inc.), AAMP (dilution, 1:1,000; cat. no. 21220-1-AP; ProteinTech Group, Inc.) and GAPDH (dilution, 1:10,000; cat. no. ab9385; Abcam) at $4^{\circ} \mathrm{C}$ overnight. Following incubating with the primary antibody, membranes were incubated with secondary antibodies [horseradish peroxidase-conjugated goat anti rabbit $\operatorname{IgG}(1: 5,000$; cat. no. ab6721) or goat anti mouse $\operatorname{IgG}(1: 2,000$; cat. no. ab6789; Abcam)] for $1.5 \mathrm{~h}$ at room temperature, which was performed in TBS-Tween solution containing 5\% skimmed milk. ECL chemiluminescence (Thermo Fisher Scientific, Inc.) was followed by exposure and development. ImageJ software (version 1.46; National Institutes of Health) was used for analysis.

Immunofluorescence. In brief, A10 cells were seeded into 24-well plates for $24 \mathrm{~h}$ and fixed with $4 \%$ paraformaldehyde at $4{ }^{\circ} \mathrm{C}$ for $30 \mathrm{~min}$, followed by permeabilization with $0.5 \%$ Triton X-100 and blocking with 5\% bovine serum albumin (Sigma-Aldrich; Merck KGaA) at room temperature for $30 \mathrm{~min}$. Samples were incubated with primary antibodies against SRF (dilution, 1:200; cat. no. 16821-1-AP; ProteinTech Group, Inc.) and AAMP (dilution, 1:200; cat. no. 21220-1-AP; ProteinTech Group, Inc.) overnight at $4^{\circ} \mathrm{C}$. Samples were subsequently washed with PBS and incubated with secondary 

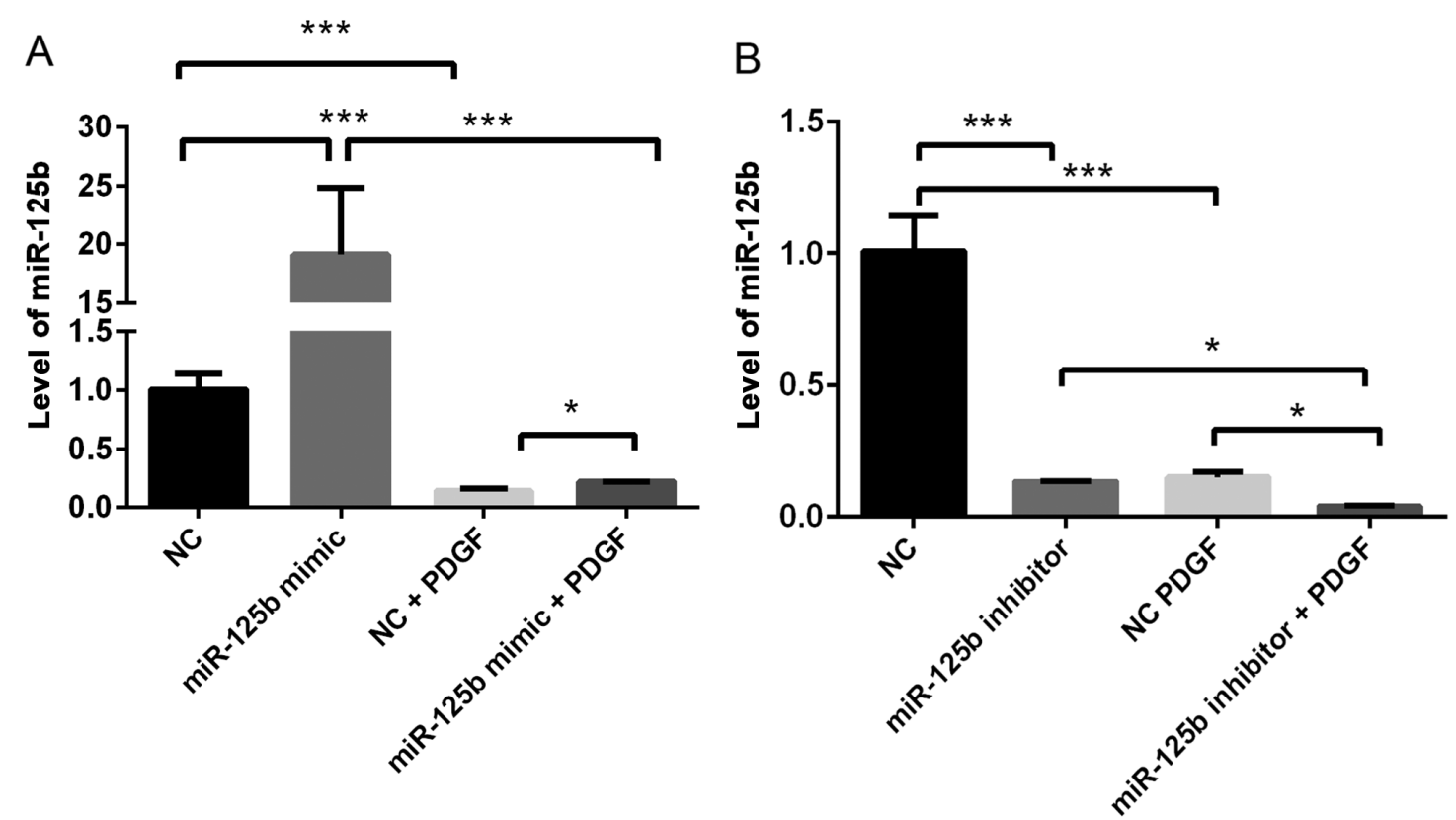

Figure 1. PDGF-BB inhibits miR-125b expression in VSMCs. (A) Treatment with PDGF-BB decreases miR-125b expression, the effects of which are reversed following transfection of A10 cells with miR-125b mimic. (B) Treatment with PDGF-BB decreases miR-125b expression, the effects of which are reversed following transfection of A10 cells with miR-125b inhibitor. Data are presented as the mean \pm standard deviation. One-way analysis of variance and the Tukey's post hoc test were used. ${ }^{*} \mathrm{P}<0.05,{ }^{* * * *} \mathrm{P}<0.001$. PDGF-BB, platelet-derived growth factor-BB; miR, microRNA; VSMCs, vascular smooth muscle cells; NC, negative control.

antibodies for $1 \mathrm{~h}$ at room temperature, prior to re-washing. Nuclei were stained with $15 \mu$ l DAPI (Sigma-Aldrich; Merck $\mathrm{KGaA}$ ) for $2 \mathrm{~min}$ at room temperature, prior to being observed under a fluorescence microscope (magnification, x200; Carl Zeiss AG).

Statistical analysis. Statistical analysis was performed using SPSS 20.0 software (IBM Corp.). All experiments were performed for three repeats excluding for Western blotting, which was only conducted once. Data are presented as the mean \pm standard deviation. Paired Student's t-test was used to compare differences between two groups, while one-way analysis of variance and the Tukey's post hoc test were used to compare differences among multiple groups. $\mathrm{P}<0.05$ was considered to indicate a statistically significant difference.

\section{Results}

PDGF-BB negatively regulates miR-125b expression in VSMCs. To determine the effect of PDGF-BB on miR-125b expression, miR-125b expression levels in VSMCs with or without PDGF-BB treatment were compared. As presented in Fig. 1, treatment with PDGF-BB significantly decreased miR-125b expression in VSMCs $(\sim 80 \%$; $\mathrm{P}=0.0004)$. Notably, overexpression of miR-125b significantly rescued miR-125b expression following treatment with PDGF-BB ( $40 \%$ upregulation; $\mathrm{P}=0.03$ ).

miR-125b inhibits the proliferation of VSMCs. To investigate the role of $\mathrm{miR}-125 \mathrm{~b}$ on the proliferation of VSMCs, the CCK- 8 and BrdU assays were performed. The results of the CCK- 8 assay indicated that the OD value was decreased in the miR-125b mimic group compared with the
$\mathrm{NC}$ group $(\mathrm{P}<0.001$; Fig. 2A); therefore, the overexpression of miR-125b inhibited the proliferation of VSMCs. Furthermore, the results of the BrdU assay demonstrated that transfection with miR-125b mimic significantly decreased the OD value at day 4 ( 40\%), compared with the $\mathrm{NC}$ group ( $\mathrm{P}<0.001$; Fig. $2 \mathrm{~B})$. Taken together, these results suggested that miR-125b inhibits cell proliferation.

miR-125b inhibits the migration of VSMCs. To investigate the role of miR-125b on the migration of VSMCs, Transwell migration and wound healing assays were performed. The results of the Transwell assay demonstrated that the number of migratory cells significantly decreased in the miR-125b mimic group compared with the $\mathrm{NC}$ group $(\mathrm{P}<0.05$; Fig. $3 \mathrm{~A})$. As presented in Fig. 3B, there were few differences between the NC and miR-125b mimic groups after $24 \mathrm{~h}$. However, following $48 \mathrm{~h}$ of incubation, the cells had almost completely migrated into the wound in the NC group, while the gaps remained visible in the miR-125b mimic group. Following normalization, the migratory ability in the miR-125b mimic group was $\sim 88 \%$ of that in the NC group $(\mathrm{P}<0.05)$.

AAMP and SRF are targets of miR-125b in VSMCs. The present study investigated the association between miR-125b expression and AAMP and SRF expression. The results demonstrated that the protein expression levels of AAMP and SRF were significantly downregulated following transfection with miR-125b mimic, the effects of which were reversed following transfection with miR-125b inhibitor $(\mathrm{P}<0.05$; Fig. $4 \mathrm{~A}$ and $\mathrm{B})$. Immunofluorescence analysis demonstrated that the miR-125b mimic-induced downregulation of AAMP and SRF was very slight, while miR-125b inhibitor-induced upregulation of AAMP and SRF expression levels was significant (Fig. 4C and D). 

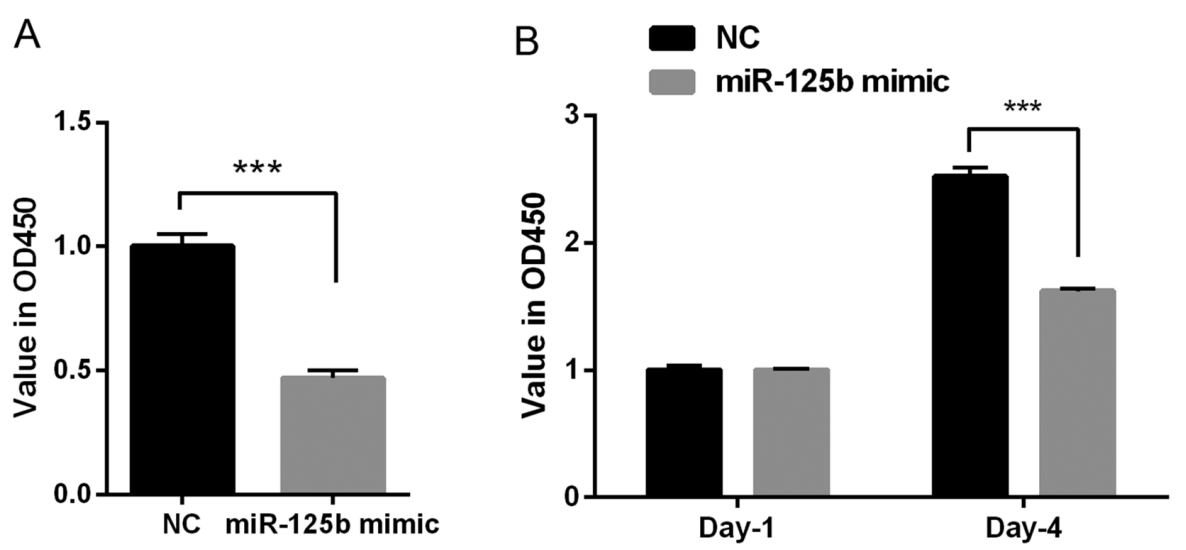

Figure 2. miR-125b inhibits the proliferation of VSMCs. (A) Cell Counting Kit-8 assay indicated that transfection with miR-125b mimic decreased the OD value at a wavelength of $450 \mathrm{~nm}$, which is approximately half of the NC group. (B) The BrdU assay demonstrated that transfection with miR-125b mimic significantly inhibited the OD value at day 4. Data are presented as the mean \pm standard deviation. Paired Students' t-test was used. ${ }^{* * *} \mathrm{P}<0.001$. miR, microRNA; VSMCs, vascular smooth muscle cells; OD, optical density; NC, negative control; OD, optical density; NC, negative control.

A
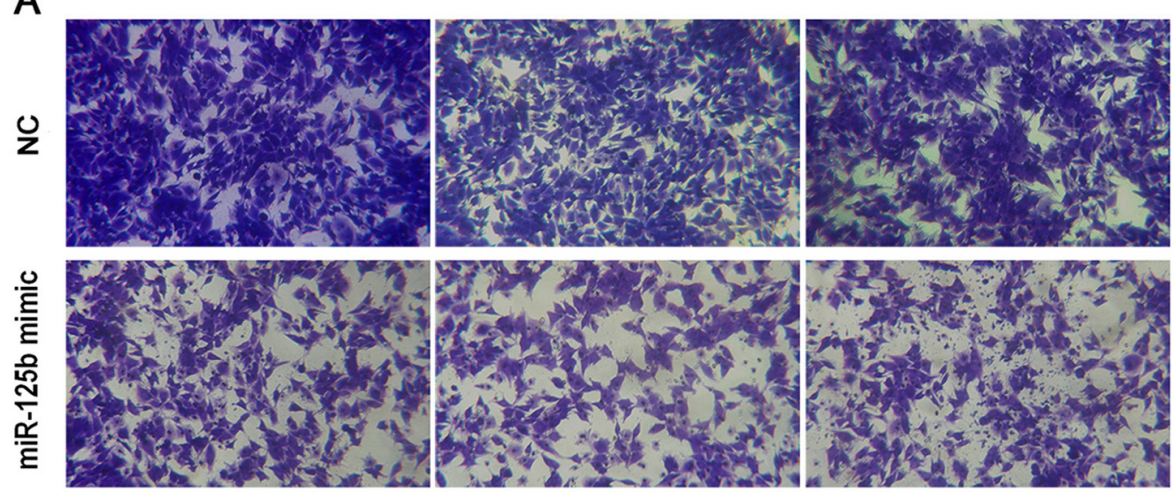

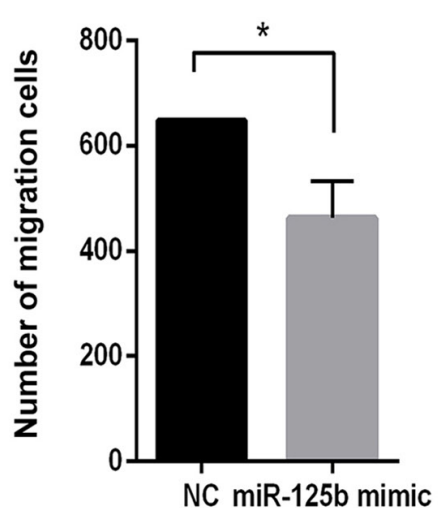

NC miR-125b mimic
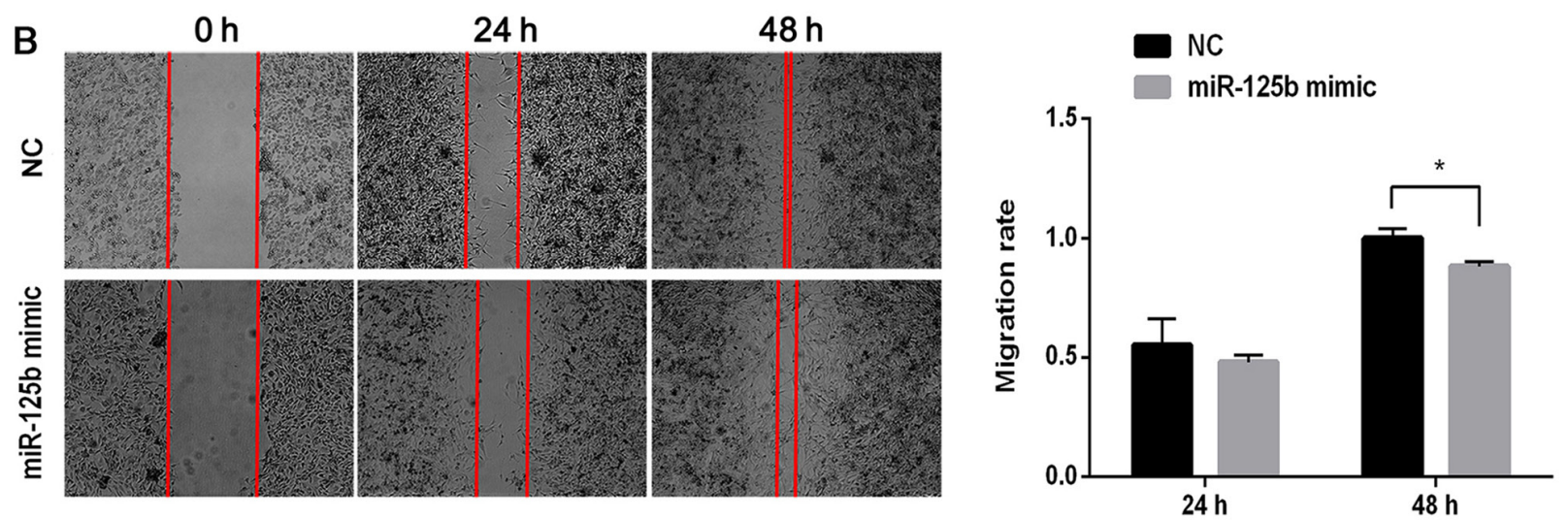

Figure 3. miR-125b inhibits the migratory ability of VSMCs. (A) Transwell migration assay demonstrated that cells migrated in the miR-125b mimic group and NC group. Images presented from three different fields. (B) Wound healing assay was performed to assess the migratory ability of VSMCs in the miR-125b mimic group and NC group at 24 and $48 \mathrm{~h}$. Data are presented as the mean \pm standard deviation. Paired Student's t-test was used. " $\mathrm{P}<0.05$. miR, microRNA; VSMCs, vascular smooth muscle cells; NC, negative control.

\section{Discussion}

Lower extremity ASO is induced by atherosclerotic thrombosis, which causes narrowing or occlusion of the lower extremity arteries, and the persistent ischemia eventually leads to tissue necrosis (9). The prevalence of ASO increases with age, which affects $\sim 8-10 \%$ of people $>65$ years and $\sim 20 \%$ of people $>80$ years (9). ASO is initiated following the accumulation of lipoproteins in the endothelium due to the abnormal metabolism of glucose and lipid $(1,10)$. This in turn results in a series of intracellular reactions, which eventually induces vascular endothelial damage and the monocyte/macrophage-induced inflammatory response (11). Inflammatory cells and factors regulate the function of VSMCs, including cell proliferation, migration, differentiation and matrix secretion (11). VSMCs are the major cellular 

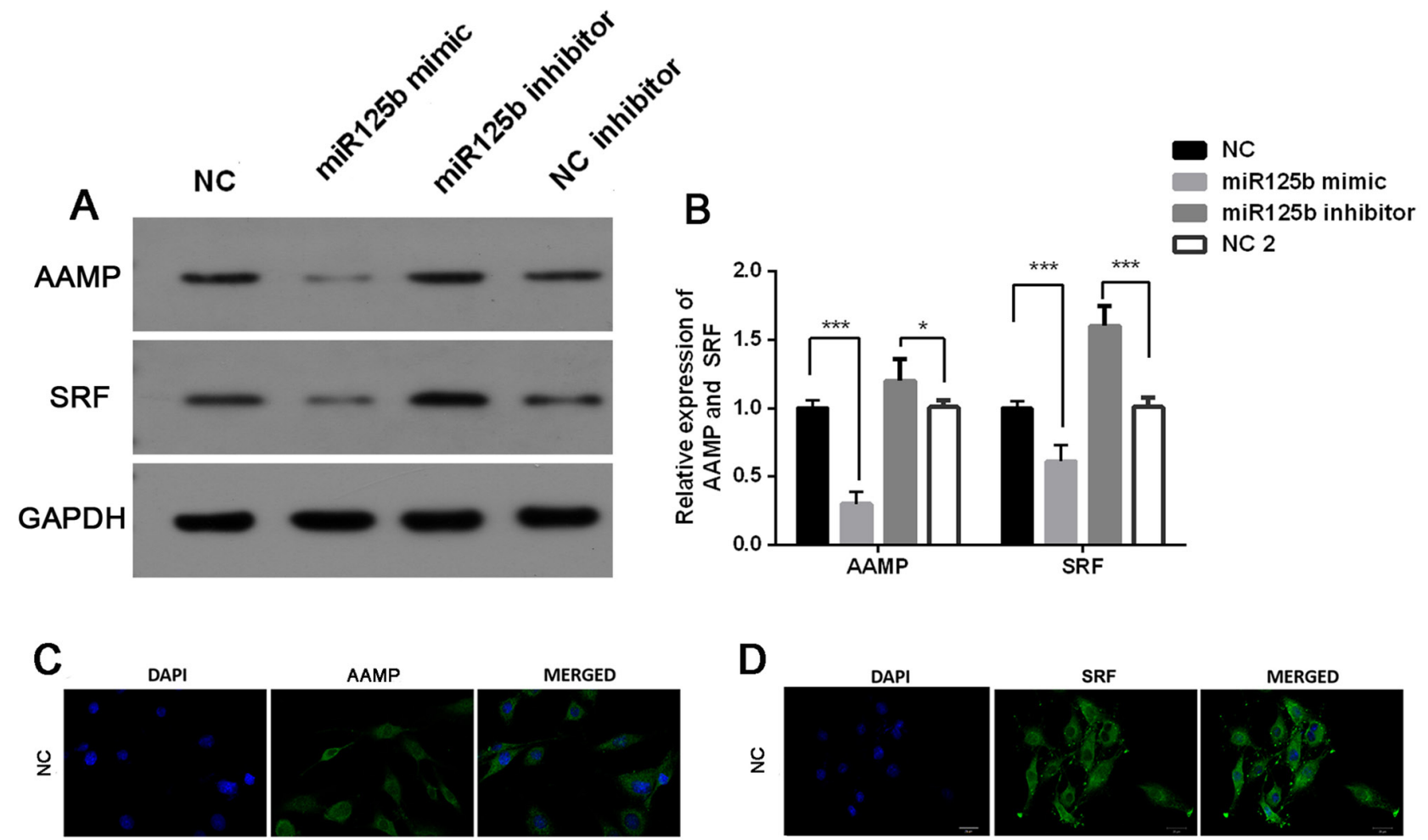

MERGED
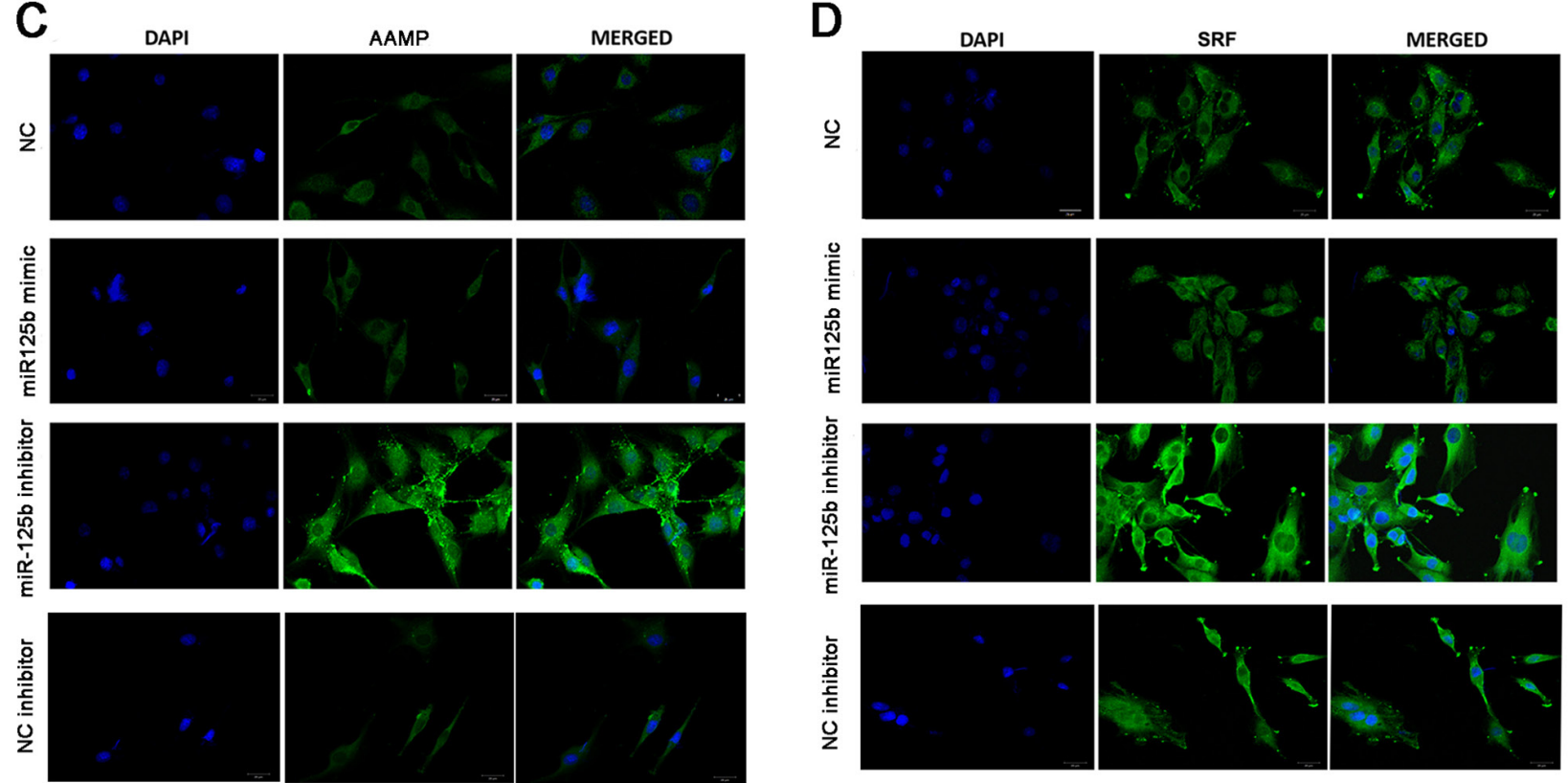

Figure 4. miR-125b negatively regulates AAMP and SRF. (A) Western blot analysis was performed to detect the protein expression levels of AAMP and SRF. (B) Semi-quantification of AAMP and SRF protein expression levels. Immunofluorescence assay was performed to detect (C) AAMP and (D) SRF expression. Data are presented as the mean \pm standard deviation. One-way analysis of variance and the Tukey's post hoc test were used. ${ }^{*} \mathrm{P}<0.05,{ }^{* * * *} \mathrm{P}<0.001$. miR, microRNA; AAMP, angio-associated migratory cell protein; SRF, serum response factor; NC, negative control.

components that make up the middle layer of the arteries and are involved in the process of vascular diseases. At present, it is believed that the excessive proliferation and migration of VSMCs is one of the main causes of ASO, and it is also the main cause of restenosis following interventional treatment (10). Therefore, the prevention and relief of dysfunction of VSMCs are important for ASO treatment.

PDGF-BB derived from platelets is considered as a mediator of VSMC dysfunction and restenosis (12). PDGF-BB is a well-known atherosclerosis promotional factor (13). In the present study, PDGF-BB was used to imitate ASO. The effect of PDGF-BB treatment on miR-125b expression in VSMCs was investigated. The results demonstrated that miR-125b expression was significantly decreased following treatment with PDGF-BB, the effects of which were reversed following transfection with the miR-125b mimic. The present study used PDGF-BB to treat cells to determine the effect of the miR-125b mimic on the migratory and proliferative abilities of VSMCs. The CCK- 8 and BrdU assays were performed to assess cell proliferation following treatment with PDGF-BB and transfection with miR-125b mimic. The results demonstrated that miR-125b mimic significantly inhibited cell proliferation. Furthermore, the results of the Transwell and wound healing assays demonstrated that miR-125b mimic decreased cell migration. Taken together, these results suggested that miR-125b serves an important role in ASO, which is consistent with previous findings (8).

Previous studies have demonstrated that miR-125b expression is downregulated in a variety of tumor cells, including hepatocellular carcinoma, breast cancer, ovarian 
tumors, bladder cancer and leukemia (14-18). Downregulation of miR-125b enhances the cell proliferative and invasive abilities. In ASO, miR-125b is one of the miRNAs that is significantly downregulated (7). It has been suggested that miR-125b can inhibit the proliferation and migration of VSMCs, and the molecular mechanisms are associated with the regulation of SRF or myosin $1 \mathrm{E}$, respectively $(7,19)$. However, miRNAs and their targets are complex and diverse (20). There is no clear evidence that miR-125b affects AAMP. AAMP is highly expressed in cancer cells, and its high expression is associated with the poor prognosis of patients with cancer (21). In the present study, miR-125b mimic and inhibitor were used to assess the effect of miR-125b expression levels on AAMP and SRF expression. Western blot analysis demonstrated that the protein expression levels of AAMP and SRF were significantly decreased following transfection with miR-125b mimic, the effects of which were reversed following transfection with the miR-125b inhibitor. The results of the immunofluorescence assay were consistent with these findings.

The results of the present study demonstrated that PDGF-BB was a negative factor of miR-125b expression in VSMCs. Furthermore, transfection with miR-125b mimic increased miR-125b expression in VSMCs, and promoted the cell proliferative and migratory abilities following treatment with PDGF-BB. miR-125b mimic and miR-125b inhibitor inhibited and induced AAMP and SRF expression, respectively. However, further studies are required to determine whether AAMP and SRF are target genes of miR-125b, and whether miR-125b has other target genes. Taken together, the results of the present study suggested that miR-125b is a potential therapeutic strategy for preventing the progression of ASO.

\section{Acknowledgements}

Not applicable.

\section{Funding}

The present study was supported by the Major Natural Science Project of Universities in Anhui Province (grant no. KJ2016SD38), the Key Project of Natural Science Foundation of Bengbu Medical College in Anhui Province (grant no. BYKY1862ZD) and the Scientific and technological research project of Anhui Province (grant no. 201904a07020020).

\section{Availability of data and materials}

The datasets used and/or analyzed during the current study are available from the corresponding author on reasonable request.

\section{Authors' contributions}

XW and YG designed the study. XW, SC, CY and ZN, YS, RL and ZG performed the experiments. SC, CY, ZN and YS performed the statistical assessments. XW, SC and YG wrote the manuscript. XW, SC and YG confirm the authenticity of all the raw data. All authors read and approved the final manuscript.

\section{Ethics approval and consent to participate}

Not applicable.

\section{Patient consent for publication}

Not applicable.

\section{Competing interests}

The authors declare that they have no competing interests.

\section{References}

1. Howard MD, Hood ED, Zern B, Shuvaev VV, Grosser T and Muzykantov VR: Nanocarriers for vascular delivery of anti-inflammatory agents. Annu Rev Pharmacol Toxicol 54: 205-226, 2014.

2. Diehm C, Allenberg JR, Pittrow D, Mahn M, Tepohl G, Haberl RL, Darius H, Burghaus I and Trampisch HJ; German Epidemiological Trial on Ankle Brachial Index Study Group: Mortality and vascular morbidity in older adults with asymptomatic versus symptomatic peripheral artery disease. Circulation 120: 2053-2061, 2009.

3. Doran AC, Meller N and McNamara CA: Role of smooth muscle cells in the initiation and early progression of atherosclerosis. Arterioscler Thromb Vasc Biol 28: 812-819, 2018.

4. Woo CC, Liu W, Lin XY, Dorajoo R, Lee KW, Richards AM, Lee CN, Wongsurawat T, Nookaew I and Sorokin V: The interaction between $30 \mathrm{~b}-5 \mathrm{p}$ miRNA and MBNL1 mRNA is involved in vascular smooth muscle cell differentiation in patients with coronary atherosclerosis. Int J Mol Sci 21: 11, 2019.

5. Kumar S, Kim CW, Simmons RD and Jo H: Role of flow-sensitive microRNAs in endothelial dysfunction and atherosclerosis: Mechanosensitive athero-miRs. Arterioscler Thromb Vasc Biol 34: 2206-2216, 2014.

6. Boucher JM, Peterson SM, Urs S, Zhang C and Liaw L: The miR-143/145 cluster is a novel transcriptional target of Jagged-1/Notch signaling in vascular smooth muscle cells. J Biol Chem 286: 28312-28321, 2011.

7. Chen Z, Wang M, Huang K, He Q, Li H and Chang G: MicroRNA-125b affects vascular smooth muscle cell function by targeting serum response factor. Cell Physiol Biochem 46: 1566-1580, 2018.

8. Livak KJ and Schmittgen TD: Analysis of relative gene expression data using real-time quantitative PCR and the 2(-Delta Delta C(T)) method. Methods 25: 402-408, 2001.

9. Warnecke-Eberz U, Chon SH, Hölscher AH, Drebber U and Bollschweiler E: Exosomal onco-miRs from serum of patients with adenocarcinoma of the esophagus: Comparison of miRNA profiles of exosomes and matching tumor. Tumour Biol 36: 4643-4653, 2015.

10. Johnson RC, Leopold JA and Loscalzo J: Vascular calcification: Pathobiological mechanisms and clinical implications. Circ Res 99: 1044-1059, 2006.

11. Furukawa K, Abumiya T, Sakai K, Hirano M, Osanai T, Shichinohe H, Nakayama N, Kazumata K, Hida K and Houkin K: Increased blood viscosity in ischemic stroke patients with small artery occlusion measured by an electromagnetic spinning sphere viscometer. J Stroke Cerebrovasc Dis 25: 2762-2769, 2016.

12. Togliatto G, Dentelli P, Rosso A, Lombardo G, Gili M, Gallo S, Gai C, Solini A, Camussi G and Brizzi MF: PDGF-BB carried by endothelial cell-derived extracellular vesicles reduces vascular smooth muscle cell apoptosis in diabetes. Diabetes 67: 704-716, 2018.

13. Bennett MR, Sinha S and Owens GK: Vascular smooth muscle cells in atherosclerosis. Circ Res 118: 692-702, 2016.

14. Chapiro E, Russell LJ, Struski S, Cavé H, Radford-Weiss I, Valle VD, Lachenaud J, Brousset P, Bernard OA, Harrison CJ and Nguyen-Khac F: A new recurrent translocation $t(11 ; 14)$ (q24;q32) involving IGH@ and miR-125b-1 in B-cell progenitor acute lymphoblastic leukemia. Leukemia 24: 1362-1364, 2010. 
15. Guan Y, Yao H, Zheng Z, Qiu G and Sun K: miR-125b targets BCL3 and suppresses ovarian cancer proliferation. Int J Cancer 128: 2274-2283, 2011.

16. Hirsch HA, Iliopoulos D, Joshi A, Zhang Y, Jaeger SA, Bulyk M, Tsichlis PN, Liu XS and Struhl K: A transcriptional signature and common gene networks link cancer with lipid metabolism and diverse human diseases. Cancer Cell 17: 348-361, 2010.

17. Huang L, Luo J, Cai Q, Pan Q, Zeng H, Guo Z, Dong W, Huang J and Lin T: MicroRNA-125b suppresses the development of bladder cancer by targeting E2F3. Int J Cancer 128: 1758-1769, 2011.

18. Rajabi H, Jin C, Ahmad R, McClary C, Joshi MD and Kufe D: MUCIN 1 oncoprotein expression is suppressed by the miR-125b oncomir. Genes Cancer 1: 62-68, 2010.
19. Wang D, Gao B, Yue J, Liu F, Liu Y, Fu W and Si Y: Exosomes from mesenchymal stem cells expressing miR-125b inhibit neointimal hyperplasia via myosin IE. J Cell Mol Med 23: 1528-1540, 2019.

20. Liu B, Li J and Cairns MJ: Identifying miRNAs, targets and functions. Brief Bioinform 15: 1-19, 2014.

21. Yao S, Shi F, Wang Y, Sun X, Sun W, Zhang Y, Liu X, Liu X and Su L: Angio-associated migratory cell protein interacts with epidermal growth factor receptor and enhances proliferation and drug resistance in human non-small cell lung cancer cells. Cell Signal 61: 10-19, 2019. 